\title{
Children who participated in school extracurricular activities were less likely to drop out or to have been arrested
}

\author{
Mahoney JL. School extracurricular activity participation as a moderator in the development of antisocial patterns \\ Child Dev 2000 Mar-Apr;71:502-16.
}

\author{
QUESTION: Does child and adolescent participation in school extracurricular activities \\ moderate the effect of risk factor patterns of antisocial behaviour from childhood to \\ young adulthood?
}

\section{Design}

Cohort study with follow up to 24 years of age (Carolina Longitudinal Study).

\section{Setting}

7 public schools in southeastern US.

\section{Participants}

695 children (mean age 12 y, 52\% girls, 25\% AfricanAmerican) initially interviewed in the 4 th (mean age 10 y) or 7 th grade (mean age 13 y). $93 \%$ were followed to 24 years of age.

\section{Assessment of risk factors}

Age, sex, race, and socioeconomic status (SES) were determined at the initial interview. At each annual assessment participants and teachers completed the Interpersonal Competence Scale (including components to measure aggression, popularity, and academic competence). Maturational status was rated by interviewers at each annual interview. At the beginning of the study, cluster analysis was used to identify 4 configurations that were homogeneous for behaviour and academic performance risk factors. Configuration 1 (C1) was characterised by children who were highly competent in all domains; C2 children were similar to $\mathrm{C} 1$ except for more normative ratings of physical maturation and SES below the mean; C3 children had moderately low academic competence, popularity, and SES and moderately high levels of aggression; and C4 children were characterised by a multiple risk profile with few or no assets. School extracurricular activity was determined using school yearbooks.

\section{Main outcome measures}

Early school drop out and criminal offending.

\section{Main results}

$16 \%$ of the participants were early school drop outs, $12 \%$ were arrested as young adults, and $6 \%$ had both outcomes. Rates of school drop outs and arrests were concentrated in C3 and C4 children. Rates and patterns were similar for boys and girls. Children who participated in school extracurricular activities, were less likely to drop out or be arrested at follow up (independent of risk classification) than those who did not participate.

C3 and C4 boys not participating in any school extracurricular activities were 2.5 times more likely than expected to drop out followed by crime. Nonparticipating C4 girls were 4 times more likely than expected to drop out early and 1.5 times more likely to drop out and then have an arrest. C4 girls who participated in extracurricular activities experienced school drop out and arrest less often than expected based on their high risk profile.

\section{Conclusions}

Children who participated in school extracurricular activities were less likely to drop out of school as adolescents or to be arrested as young adults than were similar children who were not involved.

\section{COMMENTARY}

Much is known about the development of antisocial behaviour across childhood into young adulthood. But while we have an excellent grasp of the risk factors cumulatively leading to disruptive behaviour disorders and delinquency, we have an incomplete picture of the role of protective factors in this process. One question the clinician faces in the case of antisocial behaviour is: what can I do to offset the gradual accumulation of risk in a particular youngster, knowing that prevention tends to be more effective than treatment of conduct disorders, and knowing that some of the risk factors (such as poverty) are not influenced by clinical interventions? Any longitudinal study, especially one extending over 20 years, is a welcome addition to our scientific database, especially one with a $93 \%$ retention rate of the original participants. This study by Mahoney suggests at least 1 recommendation that can be made to parents seeking preventive intervention for children at risk. If this child is somewhere between 10 and 14 years of age, and you see patterns of high risk, encourage extracurricular participation.

Why can we be positive that such a recommendation is sound? After all, this study does not describe a randomised controlled trial. Could it not be that the children who are active in extracurricular activities, are more competent at baseline, have higher motivation to participate, or have families who support them more? This study shows that an individual's competence and participation in extracurricular activities are independent of each other. We can be reasonably sure of this because of the way competence and participation were measured. Furthermore, the fact that the introduction of extracurricular activities in children with high risk profiles leads to a reduction in antisocial outcomes later in life also is compatible with this view. In other words, participation seems to protect individuals at high risk and is not simply another marker of competence at baseline.

But if the effect is statistically significant, is it clinically significant? As it currently stands, the effects shown are modest. They do not suggest that this single intervention will prevent bad outcomes. But knowing the development of antisocial behaviour, we should happily welcome any addition to our clinical armamentarium, especially if it allows us to focus on the positive competence of individuals, independent of whatever risks and deficits they may have

Hans Steiner, MD Stanford University School of Medicine Stanford, California, USA
Source of funding: National Institute of Mental Health.

For correspondence: Dr J L Mahoney, Department of University, PO Box 208205, New Haven, CT 06520, USA 